\title{
Exponential Stability of Compactly Coupled Wave Equations with Delay Terms in the Boundary Feedbacks
}

\author{
Salah-Eddine Rebiai $\left.{ }^{(}\right)$and Fatima Zohra Sidi Ali \\ LTM, Department of Mathematics, Faculty of Sciences, \\ University of Batna, 05000 Batna, Algeria \\ rebiai@hotmail.com
}

\begin{abstract}
We consider a linear system of compactly coupled wave equations with Neumann feedback controllers that contain delay terms. First, we prove under some assumptions that the closed-loop system generates a $C_{0}$-semigroup of contractions on an appropriate Hilbert space. Then, under further assumptions, we show that the closed-loop system is exponentially stable. This result is obtained by introducing a suitable energy function and by using an observability estimate.
\end{abstract}

Keywords: Coupled wave equations · Time delays · Boundary stabilization

\section{Introduction}

In $[1,2]$, Datko et al. presented examples of infinite-dimensional second-order systems that become unstable when arbitrary small time delays occur in the damping.

$\mathrm{Xu}$ et al. established in [9] sufficient conditions that guarantee the exponential stability of the one-dimensional wave equation with a delay term in the boundary feedback. Nicaise and Pignotti [6] extended this result to the multi-dimensional wave equation with a delay term in the boundary or internal feedbacks. The same type of result was obtained by Nicaise and Rebiai [7] for the Schrödinger equation.

Motivated by the references $[3,5,6,9]$, we investigate in this paper the problem of exponential stability for a linear system of compactly coupled wave equations with delay terms in the boundary feedbacks.

Let $\Omega$ be an open bounded domain of $\mathbb{R}^{n}$ with a boundary $\Gamma$ of class $C^{2}$ which consists of two non-empty parts $\Gamma_{1}$ and $\Gamma_{2}$ such that $\overline{\Gamma_{1}} \cap \overline{\Gamma_{2}}=\emptyset$. Furthermore, assume that there exists a real vector field $h \in\left(C^{2}(\bar{\Omega})\right)^{n}$ such that:

(H.1) The Jacobian matrix $J$ of $h$ satisfies

$$
\int_{\Omega} J(x) \zeta(x) \cdot \zeta(x) d \Omega \geq c \int_{\Omega}|\zeta(x)|^{2} d \Omega,
$$

for some constant $c>0$ and for all $\zeta \in L^{2}\left(\Omega ; \mathbb{R}^{n}\right)$, 
$(H .2) h(x) . \nu(x) \leq 0$ on $\Gamma_{1}$,

where $\nu$ is the unit normal on $\Gamma$ pointing towards the exterior of $\Omega$.

Consider the following coupled system of two wave equations with delay terms in the boundary conditions:

$$
\begin{array}{lr}
\frac{\partial^{2} u(x, t)}{\partial t^{2}}-\Delta u(x, t)+l(u(x, t)-v(x, t))=0 & \text { in } \Omega \times(0,+\infty), \\
\frac{\partial^{2} v(x, t)}{\partial t^{2}}-\Delta v(x, t)+l(v(x, t)-u(x, t))=0 & \text { in } \Omega \times(0,+\infty), \\
u(x, 0)=u_{0}(x), \frac{\partial u(x, 0)}{\partial t}=u_{1}(x) & \text { in } \Omega, \\
v(x, 0)=v_{0}(x), \frac{\partial v(x, 0)}{\partial t}=v_{1}(x) & \text { in } \Omega, \\
u(x, t)=v(x, t)=0 & \text { on } \Gamma_{1} \times(0,+\infty), \\
\frac{\partial u(x, t)}{\partial \nu}=-\alpha_{1} \frac{\partial u(x, t)}{\partial t}-\alpha_{2} \frac{\partial u(x, t-\tau)}{\partial t} & \text { on } \Gamma_{2} \times(0,+\infty), \\
\frac{\partial v(x, t)}{\partial \nu}=-\beta_{1} \frac{\partial v(x, t)}{\partial t}-\beta_{2} \frac{\partial v(x, t-\tau)}{\partial t} & \text { on } \Gamma_{2} \times(0,+\infty), \\
\frac{\partial u(x, t-\tau)}{\partial t}=g(x, t-\tau) & \text { on } \Gamma_{2} \times(0, \tau), \\
\frac{\partial v(x, t-\tau)}{\partial t}=h(x, t-\tau) & \text { on } \Gamma_{2} \times(0, \tau) .
\end{array}
$$

Physically, $u$ and $v$ may represent the displacements of two vibratings objects measured from their equilibrium positions, the coupling terms $\pm l(u-v)$ are the distributed springs linking the two vibrating objects. $l, \alpha_{1}, \alpha_{2}, \beta_{1}, \beta_{2}$ are positive constants, $\tau$ is the time delay, $u_{0}, u_{1}, v_{0}, v_{1}, g$ and $h$ are the initial data.

It is well known that in the absence of delay (i.e. $\alpha_{2}=\beta_{2}=0$ ), the solution of (1)-(9) with $\alpha_{1}$ and $\beta_{1}$ positive, decays exponentially to zero in the energy space $H_{\Gamma_{1}}^{1}(\Omega) \times L^{2}(\Omega) \times H_{\Gamma_{1}}^{1}(\Omega) \times L^{2}(\Omega)$ (see [5] and [3]).

The purpose of this paper is to investigate the uniform exponential stability of system (1)-(9) in the case where all the boundary damping coefficients $\alpha_{1}, \alpha_{2}, \beta_{1}$ and $\beta_{2}$ are positive. To this end, assume as in [6] that

$$
\alpha_{1}>\alpha_{2}, \beta_{1}>\beta_{2}
$$

and define the energy of a solution of (1)-(9) by

$$
\begin{aligned}
& E(t)=\frac{1}{2} \int_{\Omega}\left[|\nabla u(x, t)|^{2}+\left|\frac{\partial u(x, t)}{\partial t}\right|^{2}+|\nabla v(x, t)|^{2}+\left|\frac{\partial v(x, t)}{\partial t}\right|^{2}+\right. \\
& \left.l|u(x, t)-v(x, t)|^{2}\right] d x+\frac{1}{2} \int_{\Gamma_{2}} \int_{0}^{1}\left[\mu\left|\frac{\partial u(x, t-\tau \rho)}{\partial t}\right|^{2}+\right. \\
& \left.\xi\left|\frac{\partial v(x, t-\tau \rho)}{\partial t}\right|^{2}\right] d \rho d \Gamma
\end{aligned}
$$


where

$$
\tau \alpha_{2}<\mu<\tau\left(2 \alpha_{1}-\alpha_{2}\right)
$$

and

$$
\tau \beta_{2}<\xi<\tau\left(2 \beta_{1}-\beta_{2}\right)
$$

We show that if $\left\{\Omega, \Gamma_{1}, \Gamma_{2}\right\}$ satisfies $(H .1)$ and (H.2), then there is an exponential decay rate for $E(t)$.The proof of this result is based on Carleman estimates for a system of coupled nonconservative hyperbolic systems established by Lasieka and Triggiani in [4] and on compactness-uniqueness arguments.

The main result of this paper can be stated as follows.

Theorem 1. Assume (H1), (H.2), (10),(12) and (13). Then there exist constants $M \geq 1$ and $\omega>0$ such that

$$
E(t) \leq M e^{-\omega t} E(0)
$$

Theorem 1 is proved in Sect. 3. In Sect.2, we study the well-posedness of system (1)-(9) using semigroup theory.

\section{Well-Posedness of System (1)-(9)}

Inspired from [6] and [7], we introduce the auxilliary variables

$$
\begin{aligned}
& y(x, \rho, t)=\frac{\partial u(x, t-\tau \rho)}{\partial t} \\
& z(x, \rho, t)=\frac{\partial v(x, t-\tau \rho)}{\partial t}
\end{aligned}
$$

With these new unknowns, system (1)-(9) is equivalent to

$$
\begin{aligned}
& \frac{\partial^{2} u(x, t)}{\partial t^{2}}-\Delta u(x, t)+l(u(x, t)-v(x, t))=0 \quad \text { in } \Omega \times(0,+\infty), \\
& \frac{\partial y(x, \rho, t)}{\partial t}+\frac{1}{\tau} \frac{\partial y(x, \rho, t)}{\partial \rho}=0 \quad \text { on } \Gamma_{2} \times(0,1) \times(0,+\infty) \text {, } \\
& \frac{\partial^{2} v(x, t)}{\partial t^{2}}-\Delta v(x, t)+l(v(x, t)-u(x, t))=0 \quad \text { in } \Omega \times(0,+\infty), \\
& \frac{\partial z(x, \rho, t)}{\partial t}+\frac{1}{\tau} \frac{\partial z(x, \rho, t)}{\partial \rho}=0 \\
& \text { on } \Gamma_{2} \times(0,1) \times(0,+\infty) \text {, } \\
& u(x, t)=v(x, t)=0 \\
& \text { on } \Gamma_{1} \times(0,+\infty) \text {, } \\
& \frac{\partial u(x, t)}{\partial \nu}=-\alpha_{1} \frac{\partial u(x, t)}{\partial t}-\alpha_{2} y(x, 1, t) \\
& \text { on } \Gamma_{2} \times(0,+\infty) \text {, } \\
& \frac{\partial v(x, t)}{\partial \nu}=-\beta_{1} \frac{\partial u(x, t)}{\partial t}-\beta_{2} z(x, 1, t) \\
& \text { on } \Gamma_{2} \times(0,+\infty) \text {, } \\
& y(x, 0, t)=\frac{\partial u(x, t)}{\partial t}, z(x, 0, t)=\frac{\partial v(x, t)}{\partial t} \\
& \text { on } \Gamma_{2} \times(0,+\infty) \text {, }
\end{aligned}
$$




$$
\begin{array}{ll}
u(x, 0)=u_{0}(x), \frac{\partial u(x, 0)}{\partial t}=u_{1}(x) & \text { in } \Omega, \\
v(x, 0)=v_{0}(x), \frac{\partial v(x, 0)}{\partial t}=v_{1}(x) & \text { in } \Omega, \\
y(x, \rho, 0)=g(x,-\tau \rho), z(x, \rho, 0)=h(x,-\tau \rho) & \text { on } \Gamma_{2} \times(0,1) .
\end{array}
$$

Denote by $\mathcal{H}$ the Hilbert space

$$
\mathcal{H}=H_{\Gamma_{1}}^{1}(\Omega) \times L^{2}(\Omega) \times L^{2}\left(\Gamma_{2} ; L^{2}(0,1)\right) \times H_{\Gamma_{1}}^{1}(\Omega) \times L^{2}(\Omega) \times L^{2}\left(\Gamma_{2} ; L^{2}(0,1)\right)
$$

where

$$
H_{\Gamma_{1}}^{1}(\Omega)=\left\{u \in H^{1}(\Omega): u=0 \text { on } \Gamma_{1}\right\}
$$

We equip $\mathcal{H}$ with the inner product

$$
\begin{aligned}
& \left.\left(\begin{array}{c}
\zeta \\
\eta \\
\theta \\
\phi \\
\chi \\
\psi
\end{array}\right) ;\left(\begin{array}{c}
\widetilde{\zeta} \\
\widetilde{\eta} \\
\widetilde{\theta} \\
\widetilde{\phi} \\
\widetilde{\chi} \\
\widetilde{\psi}
\end{array}\right)\right\rangle=\int_{\Omega}(\nabla \zeta(x) \cdot \nabla \widetilde{\zeta}(x)+\eta(x) \widetilde{\eta}(x)) d x+ \\
& \mu \int_{\Gamma_{2}} \int_{0}^{1} \theta(x, \rho) \widetilde{\theta}(x, \rho) d \rho d \Gamma+\int_{\Omega}(\nabla \phi(x) \cdot \nabla \widetilde{\phi}(x)+\chi(x) \widetilde{\chi}(x)) d x+ \\
& \xi \int_{\Gamma_{2}} \int_{0}^{1} \psi(x, \rho) \widetilde{\psi}(x, \rho) d \rho d \Gamma+l \int_{\Omega}(\zeta(x)-\phi(x))(\widetilde{\zeta}(x)-\widetilde{\phi}(x)) d x
\end{aligned}
$$

Define in $\mathcal{H}$ a linear operator $\mathcal{A}$ by

$$
\begin{aligned}
& D(\mathcal{A})=\left\{(\zeta, \eta, \theta, \phi, \chi, \psi)^{T} \in H^{2}(\Omega) \times H_{\Gamma_{1}}^{1}(\Omega) \times L^{2}\left(\Gamma_{2} ; H^{1}(0,1)\right) \times\right. \\
& H^{2}(\Omega) \times H_{\Gamma_{1}}^{1}(\Omega) \times L^{2}\left(\Gamma_{2} ; H^{1}(0,1)\right) ; \frac{\partial \zeta}{\partial \nu}=-\alpha_{1} \eta-\alpha_{2} \theta(., 1) \\
& \left.\eta=\theta(., 0) \text { on } \Gamma_{2} ; \frac{\partial \phi}{\partial \nu}=-\beta_{1} \chi-\beta_{2} \psi(., 1), \chi=\psi(., 0) \text { on } \Gamma_{2}\right\} \\
& A(\zeta, \eta, \theta, \phi, \chi, \psi)^{T}=\left(\eta, \Delta \zeta+l \phi-l \zeta,-\tau^{-1} \frac{\partial \theta}{\partial \rho}, \chi, \Delta \phi-l \phi+l \zeta,-\tau^{-1} \frac{\partial \psi}{\partial \rho}\right)^{T}
\end{aligned}
$$

Then we can rewrite (14)-(24) as an abstract Cauchy problem in $\mathcal{H}$

$$
\left\{\begin{array}{c}
\frac{d}{d t} W(t)=\mathcal{A} W(t) \\
W(0)=W_{0}
\end{array}\right.
$$

where

$$
\begin{aligned}
& W(t)=\left(u(x, t), \frac{\partial u(x, t)}{\partial t}, y(x, \rho, t), v(x, t), \frac{\partial v(x, t)}{\partial t}, z(x, \rho, t)\right)^{T}, \\
& \text { and } W_{0}=\left(u_{0}, u_{1}, g(.,-. \tau), v_{0}, v_{1}, h(.,-. \tau)\right)^{T}
\end{aligned}
$$


We verify that $\mathcal{A}$ is dissipative and that $\lambda I-\mathcal{A}$ is onto for a fixed $\lambda>0$. Thus, by the Lumer-Phillips Theorem (see for instance [8]) $\mathcal{A}$ generates a strongly continuous semigroup on $\mathcal{H}$ and consequently we have

Proposition 1. For every $W_{0} \in \mathcal{H}$, problem (27) has a unique solution $W$ whose regularity depends on the initial datum $W_{0}$ as follows:

$$
\begin{aligned}
& W(.) \in C([0,+\infty) ; \mathcal{H}) \text { if } W_{0} \in \mathcal{H} \\
& W(.) \in C^{1}([0,+\infty) ; \mathcal{H}) \cap C([0,+\infty) ; D(\mathcal{A})) \text { if } W_{0} \in D(\mathcal{A}) .
\end{aligned}
$$

\section{Proof of Theorem 1}

We prove Theorem 1 for smooth initial data. The general case follows by a standard density argument.

We proceed in several steps.

\section{Step 1.}

Differentiating $E(t)$ with respect to time, we obtain

$$
\frac{d}{d t} E(t) \leq-k \int_{\Gamma_{2}}\left\{\left|\frac{\partial u(x, t)}{\partial t}\right|^{2}+\left|\frac{\partial u(x, t-\tau)}{\partial t}\right|^{2}+\left|\frac{\partial v(x, t)}{\partial t}\right|^{2}+\left|\frac{\partial v(x, t-\tau)}{\partial t}\right|^{2}\right\} d \Gamma
$$

where

$$
k=\min \left\{\alpha_{1}-\frac{\alpha_{2}}{2}-\frac{\mu}{2 \tau}, \frac{\mu}{2 \tau}-\frac{\alpha_{2}}{2}, \beta_{1}-\frac{\beta_{2}}{2}-\frac{\xi}{2 \tau}, \frac{\xi}{2 \tau}-\frac{\beta_{2}}{2}\right\}
$$

\section{Step 2.}

We rewrite

$$
E(t)=\mathcal{E}(t)+E_{d}(t)
$$

where

$\mathcal{E}(t)=\frac{1}{2} \int_{\Omega}\left\{|\nabla u(x, t)|^{2}+\left|\frac{\partial u(x, t)}{\partial t}\right|^{2}+|\nabla v(x, t)|^{2}+\left|\frac{\partial v(x, t)}{\partial t}\right|^{2}+l|u(x, t)-v(x, t)|^{2}\right\} d x$ and

$$
E_{d}(t)=\frac{1}{2} \int_{\Gamma_{2}} \int_{0}^{1}\left\{\mu\left|\frac{\partial u(x, t-\tau \rho)}{\partial t}\right|^{2}+\xi\left|\frac{\partial v(x, t-\tau \rho)}{\partial t}\right|^{2}\right\} d \rho d \Gamma
$$

$E_{d}(t)$ can be rewritten via a change of variable as

$$
E_{d}(t)=\frac{1}{2 \tau} \int_{t}^{t+\tau} \int_{\Gamma_{2}}\left\{\mu\left|\frac{\partial u(x, s-\tau)}{\partial t}\right|^{2}+\xi\left|\frac{\partial v(x, s-\tau)}{\partial t}\right|^{2}\right\} d \Gamma d s
$$

From (29), we obtain (here and throughout the rest of the paper $C$ is some positive constant different at different occurences)

$$
E_{d}(t) \leq C \int_{0}^{T} \int_{\Gamma_{2}}\left\{\left|\frac{\partial u(x, s-\tau)}{\partial t}\right|^{2}+\left|\frac{\partial v(x, s-\tau)}{\partial t}\right|^{2}\right\} d \Gamma d s
$$

for $0 \leq t+\tau \leq T$ and $T$ large enough. 


\section{Step 3.}

From Poincaré inequality and Proposition 3.5 of [4], we have for $T$ sufficiently large and for any $\epsilon>0$

$$
\begin{gathered}
\mathcal{E}(0) \leq C \int_{0}^{T} \int_{\Gamma_{2}}\left\{\left|\frac{\partial u(x, t)}{\partial \nu}\right|^{2}+\left|\frac{\partial u(x, t)}{\partial t}\right|^{2}+\left|\frac{\partial v(x, t)}{\partial \nu}\right|^{2}+\left|\frac{\partial v(x, t)}{\partial t}\right|^{2}\right\} d \Gamma d t+ \\
C\left\{\|u\|_{L^{2}\left(0, T ; H^{1 / 2+\epsilon}(\Omega)\right)}^{2}+\|v\|_{L^{2}\left(0, T ; H^{1 / 2+\epsilon}(\Omega)\right)}^{2}\right\}
\end{gathered}
$$

Inserting the boundary conditions (6) and (7) into (31), we obtain

$$
\begin{gathered}
\mathcal{E}(0) \leq C \int_{0}^{T} \int_{\Gamma_{2}}\left\{\left|\frac{\partial u(x, t)}{\partial t}\right|^{2}+\left|\frac{\partial u(x, t-\tau)}{\partial t}\right|^{2}+\left|\frac{\partial v(x, t)}{\partial t}\right|^{2}+\left|\frac{\partial v(x, t-\tau)}{\partial t}\right|^{2}\right\} d \Gamma d t+ \\
C\left\{\|u\|_{L^{2}\left(0, T ; H^{1 / 2+\epsilon}(\Omega)\right)}^{2}+\|v\|_{L^{2}\left(0, T ; H^{1 / 2+\epsilon}(\Omega)\right)}^{2}\right\}
\end{gathered}
$$

Step 4.

Estimate (30) together with (32) yields

$$
\begin{gathered}
E(0) \leq C \int_{0}^{T} \int_{\Gamma_{2}}\left\{\left|\frac{\partial u(x, t)}{\partial t}\right|^{2}+\left|\frac{\partial u(x, t-\tau)}{\partial t}\right|^{2}+\left|\frac{\partial v(x, t)}{\partial t}\right|^{2}+\left|\frac{\partial v(x, t-\tau)}{\partial t}\right|^{2}\right\} d \Gamma d t+ \\
C\left\{\|u\|_{L^{2}\left(0, T ; H^{1 / 2+\epsilon}(\Omega)\right)}^{2}+\|v\|_{L^{2}\left(0, T ; H^{1 / 2+\epsilon}(\Omega)\right)}^{2}\right\}
\end{gathered}
$$

\section{Step 5.}

We drop the lower order terms on the right-hand side of (33) by a compactnessuniqueness argument to obtain

$$
E(0) \leq C \int_{0}^{T} \int_{\Gamma_{2}}\left\{\left|\frac{\partial u(x, t)}{\partial t}\right|^{2}+\left|\frac{\partial u(x, t-\tau)}{\partial t}\right|^{2}+\left|\frac{\partial v(x, t)}{\partial t}\right|^{2}+\left|\frac{\partial v(x, t-\tau)}{\partial t}\right|^{2}\right\} d \Gamma d t
$$

Step 6.

From (28), we have

$$
E(T)-E(0) \leq-k \int_{0}^{T} \int_{\Gamma_{2}}\left\{\left|\frac{\partial u(x, t)}{\partial t}\right|^{2}+\left|\frac{\partial u(x, t-\tau)}{\partial t}\right|^{2}+\left|\frac{\partial v(x, t)}{\partial t}\right|^{2}+\left|\frac{\partial v(x, t-\tau)}{\partial t}\right|^{2}\right\} d \Gamma d t
$$

which together with (34) leads to

$$
E(T) \leq \frac{C k^{-1}}{1+C k^{-1}} E(0)
$$

The desired conclusion follows now from (35).

\section{References}

1. Datko, R.: Not all feedback stabilized hyperbolic systems are robust with respect to small time delays in their feedbacks. SIAM J. Control Optim. 26, 697-713 (1988)

2. Datko, R., Lagnese, J., Polis, M.P.: An example on the effect of time delays in boundary feedback stabilization of wave equations. SIAM J. Control Optim. 24, 152-156 (1986) 
3. Komornik, V., Rao, B.: Boundary stabilization of compactly wave equations. Asymptotic Anal. 14, 339-359 (1997)

4. Lasiecka, I., Triggiani, R.: Carleman estimates and exact boundary controllability for a system of coupled non-conservative second-order hyperbolic equations. In: Lecture Notes in Pure and Applied Mathematics, vol. 188, pp. 215-243. Marcel Dekker, New York (1997)

5. Najafi, M., Sarhangi, G.R., Wang, H.: Stabilizability of coupled wave equations in parallel under various boundary conditions. IEEE Trans. Automat. Control 42, 1308-1312 (1997)

6. Nicaise, S., Pignotti, C.: Stability and instability results of the wave equation with a delay term in the boundary or internal feedbacks. SIAM J. Control Optim. 45, 1561-1585 (2006)

7. Nicaise, S., Rebiai, S.: Stabilization of the Schrödinger equation with a delay term in boundary feedback or internal feedback. Portugal. Math. 68, 19-39 (2011)

8. Pazy, A.: Semigroups of Linear Operators and Applications to Partial Differential Equations. Springer, New York (1983)

9. Xu, G.Q., Yung, S.P., Li, L.K.: Stabilization of wave systems with input delay in the boundary control. ESAIM Control Optim. Calc. Var. 12, 770-785 (2006) 